\title{
A Fuzzy Decision Support Method for Customer Preferences Analysis based on Choquet integral
}

\author{
Huy Quan Vu, Gang Li and Gleb Beliakov \\ School of Information Technology, Deakin University \\ 221 Burwood Highway, Vic 3125, Australia \\ Email: quan@tulip.org.au,gang.li@deakin.edu.au,gleb.beliakov@deakin.edu.au
}

\begin{abstract}
The explosion of the Web 2.0 platforms, with massive volume of user generated data, has presented many new opportunities as well as challenges for organizations in understanding consumer's behavior to support for business planning process. Feature based sentiment mining has been an emerging area in providing tools for automated opinion discovery and summarization to help business managers with achieving such goals. However, the current feature based sentiment mining systems were only able to provide some forms of sentiments summary with respect to product features, but impossible to provide insight into the decision making process of consumers. In this paper, we will present a relatively new decision support method based on Choquet Integral aggregation function, Shapley value and Interaction Index which is able to address such requirements of business managers. Using a study case of Hotel industry, we will demonstrate how this technique can be applied to effectively model the user's preference of (hotel) features. The presented method has potential to extend the practical capability of sentiment mining area, while, research findings and analysis are useful in helping business managers to define new target customers and to plan more effective marketing strategies.
\end{abstract}

\section{INTRODUCTION}

Decision making is one of the common tasks that many of us face in daily life. An action that people do naturally to support decision making process is to seek opinion from others. With the advances of Internet technology, the way that people communicate, express their view or seek opinions has been significantly changed by the online communication. With the explosion of Web 2.0 platforms such as blogs, discussion forums, peer-to-peer networks, and various other types of social media, the world has seen a revolution in social media which makes the use of social network web sites number one activity on the Web [1]. Information available on the Web has been indeed representing a potential gold-mine for companies to identify products and features which consumers are most interested in and how consumer's focus changes in response to a new advertisement campaign. Therefore, a clear overview of customer profiles has been considered as a key factor in accurate planning and decision making which are preliminary conditions for the success of today's business [2].

However, it is exactly the quality of massive volume of data that makes online information so powerful, also makes it hard to discover the sentiments contained inside. Sentiment Mining has emerged to be a powerful tool to deal with this issue by providing tools for automated opinion discovery and summarization [3]. As the matter of fact that people usually make purchasing decision for a particular product based on a number of criteria such as the product features or their own preferences, the understanding of people's opinion expressed on each product feature is crucial. Feature based sentiment mining, a branch of Sentiment Mining, has grown out of this need [4]. The technique in this area is able to provide automatic discovery and summarization of opinion from online reviews with respect to product features [5]. For instance, features of a camera that people can express opinion on include picture quality, size, battery life, price, etc. The expressed opinion can be in one of the pre-determined polarities (positive, neutral, and negative) [4] or rating inferences such as scores and stars [6]. A feature based sentiment summary system can help evaluating review comments and report the number of positive/negative comments of features or overall score of the products [3]. In this way, users can have an overall picture of what other people opinions are, without wasting time to read all available reviews.

Despite great efforts were made in this area, the current sentiment summary systems are unable to provide insight into consumer's behavior. More specifically, a customer may give low rating on the battery life and the size for a camera but still purchase it as they like its picture quality. The identification of the key features, which significantly influent customer's decision making process, can provide business managers with guidance on what features to improve and how to promote the sale of their products. Unfortunately, the current feature based sentiment summary systems are only able to provide summary of sentiment from reviews, but do not allow to effectively evaluate the importance of each feature in relation to customer's preference.

In fact, the issue of modeling consumer's behavior with respecting to multiple criteria such as product features can be formulated into a multi-criteria decision making (MCDM) problem [7], which is usually supported by techniques such as the Fuzzy Decision Support System (FDSS) [8]. For instance, Ngai and Wat developed a FDSS for risk assessment in ecommerce development [9], Grabish and Roubens applied aggregation function, a fuzzy decision support technique, to MCDM for game theory [10]. Lu et al. proposed a multicriteria group decision-making method to evaluate non-woven cosmetic product prototypes [11]. Recently, Ma et al. implemented a FDSS, called Decider, to increase the level of overall satisfaction for the final decision across the groups of 
respondents [7]. Recently, more advanced FDSS for MCDM have also been created [12]-[14]. Therefore, it would be advantageous to consider those techniques to extend the practical capability of feature based sentiment mining in helping business managers to understand the MCDM process of consumers.

In this paper, we will introduce a relatively new fuzzy decision support technique, based on an aggregation function named Choquet Integral [15] into feature based sentiment mining. This technique is able to address the requirement of business managers in utilizing sentiment mining systems to support their business planning process. Using a fuzzy measure toolbox, named fmtools, which was recently developed by Beliakov [16], we will present how FDSS can be applied to assess user preferences from product reviews collected from web sites. Our study case is an application in Tourism and Hospitality industry, particularly for Hotel industry in Singapore.

Having introduced the research motivation, the rest of the paper is organized as follows; Section II provides a brief overview of feature based sentiment mining, together with a critical review of existing works which applied FDSS into sentiment mining area. Section III introduces the concept of aggregation function together with the fmtools toolbox that we use in this study. After that, Section IV describes our data collection, experiment design, and analysis of result finding. Section V summarizes this research and provides a vision on future research directions.

\section{PRELIMINARY}

\section{A. Feature Based Sentiment Mining}

The research of feature based sentiment mining was originally formulated by $\mathrm{Hu}$ [4], and is composed of the following four subtasks:

1) Identifying product features from review comments;

2) Identifying opinion words regarding product features;

3) Determining the polarity of opinion words;

4) Polarity determination of review regarding product features.

Since then, many researchers have been trying to improve the accuracy and efficiency of existing sentiment summary systems. Some works focused on improving the discovery of product features to explore in more details the aspect on which people express their opinions [17]-[19]. Others focused on identification of opinion words [20], [21], or special words, phrases and language constructs which have impacts on opinions [22]. While some attempted to improve accuracy in determining the sentiment polarity of sensitive words [5], or review text [23], [24].

In view of practical application, a number of attempts have been made to support user decision making process by creating sentiment summary systems. Some of the systems can show the numbers of positive and negative reviews [4], [25], or display them in percentage with respect to opinions about products (cameras, mobile phones, etc.) [18], Others are designed to summarize and show the overall rating on each feature about products [26], [27]. Users are also able to visualize the sentiment scores of different products in the same figure and this makes it easy for product comparison [28].

\section{B. Fuzzy System for Sentiment Mining}

The literature of sentiment mining have witnessed a number of attempts in using fuzzy system concepts to predict sentiment. Huettner and Subasic hand-crafted a cognitive linguistic model for affection sentiments based on fuzzy logic [29]. Later on, they manually constructed a lexicon associating words with affection categories which can specify intensity (strength of affect level) and centrality (degree of relatedness to the category), then classified documents by fuzzy sets [30]. In recent works, Azrifah et al. proposed a fuzzy logic model to perform semantic classifications of customer's reviews into one of the classes: very weak, weak, moderate, very strong and strong by combinations of adjective, adverb and verb to increase the accuracy of lexicon approach [31]. Leung et al. used fuzzy string matching by regular expressions when identifying negation words [32]. In other works, the concept of aggregation function was applied in determining the orientation of opinion expressed on each feature [33]. Aggregation functions such as average and sum were also applied to calculate sentiment score of a document based on score of each term [34].

\section{Problem definition and research objectives}

In view of feature based sentiment mining applications, a major shortcoming of existing works in designing the sentiment summary systems is that the output information was highly concentrated on supporting decision making process of individuals. Organizations, companies and business managers who want to make use of these systems in business planning processes still face significant barriers. Namely, business managers would want to know what aspects of products are most concerned by consumers in order to improve and promote the sale of their products. Besides, it is important for business managers to be able to identify their target customers in designing marketing strategy, thus, they would want to know about the preference of different target customers. Such information is helpful for business managers to design marketing strategies which are more focus and effective. Existing works were not paying much attention to these aspects, and no significant attempt was made to satisfy such requirements.

For fuzzy system application, the properties of fuzzy set and aggregation function have been used in some sub-tasks of sentiment mining area. However, no specific application was found in supporting decision making process of business managers in the field of feature based sentiment mining. Besides, other properties of fuzzy measures such as Shapley value and Interaction Index have not found their application in this area yet, while their properties appear to be promising: Shapley value can be used to measure overall importance of each feature in contribution to final decision; while Interaction 
Index can measure the interaction between features in decision making process [10].

On the other hand, we noticed that a recent work by Wang et al. [27] has a similar objective to our study in terms of weighting the features to analyze reviewer's rating behavior and preferences. An assumption of their work is that the overall rating is generated based on a weighted combination of the latent ratings over all the aspects, where the weights are to model the relative emphasis that the reviewer has placed on each aspect when giving the overall rating. However, this assumption is not close to reality, where the criteria are often not independent, and positive or negative interactions may exist among them. For instance, two or more features may refer basically to the same concept, the combination of input features such as the weighted means would double count the scores of those features. Therefore, such assumption may not be able to explore fully and model accurately the decision making process of consumers.

With the aim of addressing above limitations, we present an application of a new fuzzy decision support technique into feature based sentiment mining. This can help developing better sentiment summary systems, which provide better support for business managers in their planning process. In the context of Hotel industry, the objectives of this work could be seen as following:

- Introducing a new technique into feature based sentiment summary system which is able to evaluate the importance of features in product (hotel) rating process of consumers. Reviewer's profiles such as background and travel type will also be considered and reported.

- Exploring the usage of some metrics in fuzzy system such as Shapley value and Interaction Index, and evaluate their effectiveness in sentiment mining application of hotel industry.

- Demonstrating the advantage of the presented method in modeling the multi-criteria decision making process in consideration to criteria dependence, which is more practical for real life application.

\section{MethodOLOGY}

In this section, we firstly present the concept of aggregation function and fuzzy measures, then we describe how Shapley value and Interaction Index can be computed from fuzzy measures. After that, we will provide a brief introduction to the fmtool toolbox which provides calculation functions for these metrics.

\section{A. Aggregation Functions}

Aggregation is the process of combining several inputs into a single representative output. Typically, the inputs and outputs are considered on the unit interval $[0,1]$. Formally, an aggregation function can be defined as a function with $n>1$ arguments, $f:[0,1]^{n} \rightarrow[0,1]$ with the following properties:

$$
f\left(a_{1}, a_{2}, \ldots, a_{n}\right)=0, \text { if } a_{i}=0, \forall i
$$

$$
\begin{gathered}
f\left(a_{1}, a_{2}, \ldots, a_{n}\right)=1, \text { if } a_{i}=1, \forall i \\
x \leq y \text { implies } f(x) \leq f(y), \forall x, y \in[0,1]^{n}
\end{gathered}
$$

Eq. (1a) and (1b) present the boundary conditions of this aggregation function. Eq. (1c) specifies the monotonicity condition with respect to the inputs, the partial order of vectors " $\leq$ " is understood component-wise. Besides, another property of aggregation functions is called averaging, which bounds its value by $\min (x) \leq f(x) \leq \max (x), \forall x \in[0,1]^{n}$.

Two well-known aggregation functions are the weighted arithmetic mean (WAM) and the ordered weighted averaging (OWA) operator [35], [36]. However, the disadvantage of these methods is that they are only able to take into account relative weighting of individual inputs, while the MCDM process is usually performed under the relative influence within groups (coalitions). Therefore, we use another type of aggregation function named Choquet Integral, which is defined with respect to a fuzzy measure taking into account the interplay of non-independent criteria, and can thus handle this issue [37].

\section{B. Fuzzy Measures}

The computation of Choquet integrals is based on a fuzzy measure. Here we provide an introduction to its concepts. Let set $N=\{1,2, \ldots, n\}$. A discrete fuzzy measure is a set function $v: 2^{N} \rightarrow[0,1]$ which is monotonic (i.e. $v(A) \leq v(B)$ whenever $A \subset B)$ and satisfies $v(\emptyset)=0$ and $v(N)=1$. Since subset $A \subseteq B$ can be considered as a coalition, $v(A)$ is able to represent the importance or weight of this coalition. Note that, the monotonicity condition implies that its weight does not decrease when adding new elements to a coalition.

The discrete Choquet integral with respect to a fuzzy measure $v$ is given by

$$
C_{v}(x)=\sum_{i=1}^{n} x_{i}\left[v\left(\left\{j \mid x_{j} \geq x_{i}\right\}\right)-v\left(\left\{j \mid x_{j} \geq x_{(i+1)}\right\}\right)\right]
$$

where $x_{1}, x_{2}, \ldots, x_{n}$ is a non-decreasing permutation of the input $x$, and $x_{(n+1)}=\infty$ by convention.

\section{Shapley Value}

Since the Shapley value measures the overall importance of each element in terms of its contribution to the score of each coalition, it can measure better the importance of each feature in contribution to multi-criteria decision making problem.

Let $v$ be a fuzzy measure. The Shapley index for every input $i \in N$ is

$$
\phi_{i}=\sum_{A \subseteq N /\{i\}} \frac{(n-|A|-1) !|A| !}{n !}[v(A \cup\{i\})-v(A)]
$$

The Shapley value is the vector $\phi(v)=\phi_{1}, \ldots, \phi_{n}$, and satisfies $\sum_{i=1}^{n} \phi_{i}=1$. 


\section{Interaction Index}

The Interaction Index is interpreted as the behavior of criteria in coalitions. Interaction index for every pair of input features $i, j \in N$ is defined as:

$$
\begin{array}{r}
I_{i j}=\sum_{A \subseteq N /\{i, j\}} \frac{(n-|A|-2) !|A| !}{(n-1) !}[v(A \cup\{i, j\}) \\
-v(A \cup\{i\})-v(A \cup\{j\})+v(A)]
\end{array}
$$

For any pair $i, j$, we have $I_{i j} \in[-1,1]$. If $i$ and $j$ are positively correlated, then $I_{i j}<0$. Similarly, if $i$ and $j$ are negatively correlated, then $I_{i j}>0$. This measure is more than just the interaction between pairs of criteria by themselves. Each pair is considered in the presence of all coalitions. It may be that two criteria interact positively in isolation, but in larger groups they may contribute very little. Such behavior can be measured using Interaction Index.

\section{E. FMTools}

FMTools [16] is a software package implemented in $\mathrm{C}++$ programming language for evaluating Choquet Integrals and performing operations on fuzzy measures. There are two main programs, fuzzy measure estimation and aggregation function, embedded in this toolbox.

Fuzzy measure estimation program learns the coefficients of a suitable fuzzy measure from observations by fitting Choquet integral to the data. The fitting is performed by minimising the sum of absolute deviations between the predictions and observations, using linear programming techniques. The details are given in [15], [38]. Fuzzy measure estimation program is an executable named fmfitting which is compiled for MS Windows and Linux $i 386$. It takes parameters from a configuration file. Some basic parameters for input file are $N$ (the number of input argument), $M$ (the number of data instances), data set (name of file containing empirical data), output (name of output file). Given a data set containing $M$ instances $x_{1}, x_{2}, \ldots, x_{M}$, each instance $x_{i}$ is composed by $N$ inputs $x_{i 1}, x_{i 2}, \ldots, x_{i N}$. Fuzzy measure $v$ can be estimated from this data such that the corresponding Choquet Integral $f=C_{v}$ predicts the output $y_{k}$ as close as possible in the least absolute deviation sense. The detailed description of optimization procedure for fuzzy measure estimation can be obtained from [16]. Then other values such as Shapley and Interaction Index can be computed following equations 3 and 4.

In general, the concepts of fuzzy measure and aggregation function have been utilized in some applications of decision making [10], [39]. Choquet Integral has also been used as an ordinal classifier in Journal Ranking application [40]. In this paper, we will focus on investigating the usage of Shapley value and Interaction Index in Hotel industry customer reviews analysis, which can address the shortcomings of existing feature based sentiment summary systems.

\section{Study CAse And Analysis}

\section{A. Data collection}

The hotel review data set that we used in this study is collected from TripAdvisor 1, a well-known travel review web site which has been investigated in Sentiment Mining area [27], [41]. An advantage of this web site is that each review comment contains not only review text but also direct review rating for each hotel feature as well as an overall rating. Since our study focus on evaluating the importance of hotel features rather than sentiment prediction from review text, we only extract the hotel feature rating and overall rating from each review. The ratings for the hotels are in scale from 1 (very disappointed) to 5 (very satisfied), which includes value for money (Value), hotel location (Location), quality of sleep (Sleep), quality of room (Room), room cleanliness (Cleanliness), and additional service (Service). The hotel ratings and other reviewer demographic data such as traveler types (business, family, couple) and countries of origin are extracted automatically using a professional data extraction software, called Visual Web Ripper ${ }^{2}$. The software navigated through all listed hotels in Singapore and extracted all review comments posted in year 2011. Note that, this is an automated process and some reviews did not provide rating for all 6 listed hotel features, and a number of data instances are with missing attributes. We removed all those missing data, and this left us with approximately 8790 records.

\section{B. Experiment Design}

Since our target is to study the preference and profile of consumers, we divide our data set into a number of sub-data sets according to reviewer's travel types and regions. Let us assume that people from different continents would have different backgrounds [42]. Therefore, we group the regions according to reviewer's continents of origin. It is interesting that, the majority of people are from North America, Europe, Asia and Oceania, while very few people are from South America and Africa. Thus, we only consider those four continents in this study.

Besides, we would like to evaluate the performance of Choquet Integral against typical methods such as the weighted arithmetic mean (WAM), the ordered weighted averaging (OWA) and the unrestricted linear model (LM). These experiments are carried out on the collected Hotel data set using 10-fold cross validation strategy. We use the root mean square error (RMSE) to measure the prediction error of those algorithms:

$$
R M S E=\sqrt{\frac{1}{n} \sum_{n=1}^{n}\left(y_{i}-f\left(x_{i}\right)\right)^{2}}
$$

where $y_{i}$ is the value of the $i^{t h}$ input instance to be predicted, and $f\left(x_{i}\right)$ is the predicted value of $y_{i}$.

\footnotetext{
${ }^{1}$ http://www.tripadvisor.com

${ }^{2} \mathrm{http} / / / \mathrm{www} . v i s u a l w e b r i p p e r . c o m$
} 
More importantly, we will demonstrate the advantage of Choquet Integral by analyzing the interaction index outputs between hotel features. Notice that the input data to Choquet Integral is in the range $[0,1]$, therefore, we perform normalization for all the hotel rating scores into this range before fitting them into fmfitting function.

In order to achieve the above objectives, we will perform two major experiments on the collected data set: 1) model evaluation and 2) profile study of travelers. The model evaluation experiment will be performed on Hotel features for different types of travelers to evaluate algorithm's performance. Then, the profile study of travelers case will construct a detailed profile for Hotel features preference of travelers with respect to travel groups as well as their regions of origin. The description of the sub-data sets for these analysis are shown in Table I.

TABLE I: Structure of sub-data sets for each experiment

\begin{tabular}{cccr}
\hline Study Case & Travel Type & Region & Size \\
\hline \multirow{3}{*}{1} & Business & All 4 Continents & 2579 instances \\
& Couple & All 4 Continents & 4066 instances \\
& Family & All 4 Continents & 1916 instances \\
\hline \multirow{3}{*}{ Business } & Asia & 1210 instances \\
& & Europe & 581 instances \\
& & North America & 407 instances \\
& & Oceania & 381 instances \\
\cline { 3 - 4 } 2 & Couple & Asia & 1169 instances \\
& & Europe & 1389 instances \\
& & Oceania & 1182 instances \\
& & Asia & 951 instances \\
\cline { 2 - 4 } & & Europe & 309 instances \\
& \multirow{3}{*}{ Family } & North America & 131 instances \\
& & Oceania & 525 instances \\
\hline
\end{tabular}

\section{Result Analysis}

1) Model Evaluation: In this section, we evaluate the performance of Choquet Integral (CI) against other algorithms (WAM, OWA and LM). We use the sub-data sets of the first study case for evaluation purpose. Each of the sub-data sets for different travel types (business, couple, and family) is randomly divided into 10 sets. The algorithms are applied on 9 sets and tested on 1 set, and this process is repeated 10 times according to 10 -fold cross validation strategy. The RMSE values are recorded and shown in Table II.

TABLE II: RMSE values of the evaluated algorithms.

\begin{tabular}{cccccc}
\hline \multirow{2}{*}{ Travel Type } & \multirow{2}{*}{ Regions } & LM & WAM & OWA & CI \\
\hline Business & All 4 continents & 0.0966 & 0.0966 & 0.0987 & $\underline{0.0955}$ \\
Couple & All 4 continents & 0.0928 & 0.0928 & 0.0950 & $\underline{\underline{0.0917}}$ \\
Family & All 4 continents & 0.0933 & 0.0934 & 0.0947 & $\underline{0.0931}$ \\
\hline
\end{tabular}

From Table II, OWA appears to be the algorithm with the highest RMSE values. Most importantly, Choquet Integral has outperformed all others algorithms with lowest RMSE values. This evidence supports the claim that Choquet Integral can model the input data better than other popular algorithms.

Even though, Choquet Integral outperformed other algorithms in fitting input data, it is necessary to verify the Shapley value of Choquet in evaluating the importance (weight) of feature. For this purpose, we compare the output weights of Choquet and a popular feature weighting method (WAM). In this case, the entire sub-data sets are fitted into IC and WAM, output weights of two algorithms on Hotel features for different travel groups are shown in Figure 1.

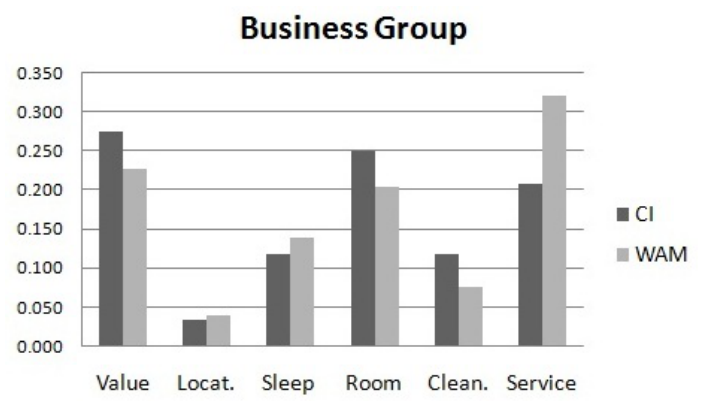

(a)

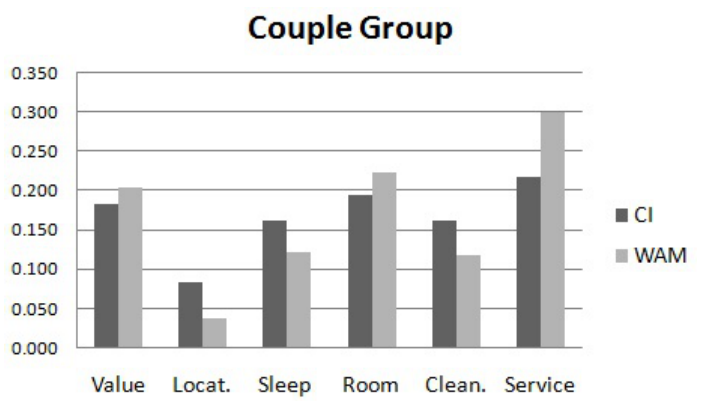

(b)

\section{Family Group}

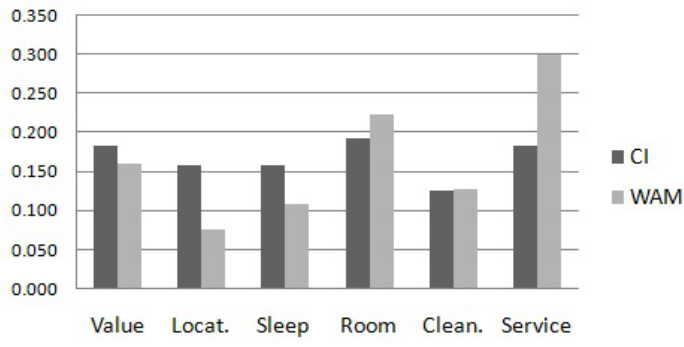

(c)

Fig. 1: Weights of Hotel Features estimated by Choquet Integral and WAM for different travel groups

From the Figure 1, we can see that there are some slightly differences between the weight of Choquet and WAM for all three data sets. However, the importance of each hotel features with respect to other features are relatively similar for both algorithms. More specifically, both Choquet and WAM evaluated features including the value, room and service to be significantly more important than other features in the case of business group and couple group. Besides, features including location, sleep and cleanliness are evaluated as slightly less important than the other features in the case of family group. These evidences indicate that Choquet Integral can preserve the relationship between feature's importance as the standard 
TABLE III: Interaction Indices between Hotel Features for different Groups of Travelers

\begin{tabular}{|c|c|c|c|c|c|c|}
\hline \multirow{2}{*}{$\frac{\text { Travel Type }}{\text { Business }}$} & \multicolumn{6}{|c|}{ Interaction Index } \\
\hline & $\begin{array}{r}\text { Value } \\
\text { Location } \\
\text { Sleep } \\
\text { Room } \\
\text { Cleanliness }\end{array}$ & $\begin{array}{l}\text { Location } \\
-0.017\end{array}$ & $\begin{array}{r}\text { Sleep } \\
-0.058 \\
0.067\end{array}$ & $\begin{array}{r}\text { Room } \\
\mathbf{0 . 3 1 7} \\
-0.058 \\
-0.100\end{array}$ & $\begin{array}{r}\text { Cleanliness } \\
-0.058 \\
0.067 \\
-0.058 \\
-0.100\end{array}$ & $\begin{array}{r}\text { Service } \\
-0.183 \\
-0.058 \\
\mathbf{0 . 1 5 0} \\
-0.058 \\
\mathbf{0 . 1 5 0}\end{array}$ \\
\hline Couple & $\begin{array}{r}\text { Value } \\
\text { Location } \\
\text { Sleep } \\
\text { Room } \\
\text { Cleanliness }\end{array}$ & $\begin{array}{r}\text { Location } \\
0.017\end{array}$ & $\begin{array}{r}\text { Sleep } \\
\mathbf{0 . 1 5 6} \\
-0.067\end{array}$ & $\begin{array}{r}\text { Room } \\
-0.094 \\
0.017 \\
0.044\end{array}$ & $\begin{array}{r}\text { Cleanliness } \\
\mathbf{0 . 1 5 6} \\
-0.067 \\
-0.039 \\
-0.122\end{array}$ & $\begin{array}{r}\text { Service } \\
-0.233 \\
0.100 \\
-0.094 \\
\mathbf{0 . 1 5 6} \\
0.072\end{array}$ \\
\hline Family & $\begin{array}{r}\text { Value } \\
\text { Location } \\
\text { Sleep } \\
\text { Room } \\
\text { Cleanliness }\end{array}$ & $\begin{array}{l}\text { Location } \\
-0.108\end{array}$ & $\begin{array}{r}\text { Sleep } \\
0.058 \\
0.017\end{array}$ & $\begin{array}{r}\text { Room } \\
\mathbf{0 . 1 4 2} \\
-0.066 \\
-0.067\end{array}$ & $\begin{array}{r}\text { Cleanliness } \\
\mathbf{0 . 1 4 2} \\
-0.067 \\
-0.067 \\
0.017\end{array}$ & $\begin{array}{r}\text { Service } \\
-0.233 \\
\mathbf{0 . 2 2 5} \\
0.058 \\
-0.025 \\
-0.025\end{array}$ \\
\hline
\end{tabular}

method WAM. Therefore, it is possible to use the weights that were generated by Choquet for feature evaluation purpose.

Analysis of Interaction Indices: Another advantage of Choquet Integral over the weighted arithmetic mean and the ordered weighted averaging operator is the ability of providing insight into the interaction between features. Here, we demonstrate such utility by analyzing the interaction indices between hotel features for different groups of travelers as shown in Table III. Note that, for a pair of features, a negative value represents a correlation or redundancy relationship, while a positive value represents a complementary effect.

From the pair-wise interaction of Hotel features in the Table III, we can see that, generally, most pairs show little interaction for all three groups of travelers. Even though, the interaction indices are expressing some interesting information as following:

- Most hotel feature pair in business groups show little interaction with each other as indicated by low interaction indices. Besides, value for money feature was found to have strong complementary effect with room quality (0.317), while, it is slightly redundant with service features. Sleep quality and cleanliness have considerably significant complementary effect with service feature as indicated by interaction index of 0.150 .

- For people traveling as couple, value for money appears to have fairly high complementary effect with sleep quality and cleanliness, whereas, it is highly redundant with service feature. Room quality is slightly correlated with cleanliness, but complement with service feature.

- Similar to couple group, value for money feature is correlating with service feature for people traveling with family as shown by the interaction index of -0.233 . In addition, value for money has significant complement effect with both the room quality and the service feature (0.142) Furthermore, it is interesting to see that location is highly compliment with service feature for this group.

2) Profile study of Travelers: The study of user profile is crucial for business managers in designing effective marketing strategies. Thus, in this study case, we construct a profile for Hotel features preference of travelers with respect to travel groups and regions of origin. The sub-data sets for study case are input into fmfitting function. The Shapley values indicating the importance of hotel features are shown in Table IV

From the Shapley value in Table IV, the hotel preference profile of people traveling to Singapore can be constructed as following:

- Business Group: People from Asia care more about the features such as value for money, room quality and services, especially, room quality was the most concerned feature. For European and Oceania travelers, their preferred features are room quality and service as indicated with high Shapley values, particularly for service, it is highly considered by Oceania people (0.367). On the other hand, business people from North American countries are considering more the value for money and sleep quality features of hotel.

- Couple Group: It is interesting to see that Asian and European people are paying high attention to value for money feature when they travel with their partners. Besides, European people are also concerning more about service feature. For North American and Oceania couples, their preferred features are room quality and service quality, especially, room quality was most important to North American people (0.303). In general, most hotel features are having certain level of influence to the overall rating of people traveling as couple as shown with relatively significant Shapley values (above 0.1).

- Family Group: The importance of value for money feature remain significant for Asian people in this group, while, European people care more about room quality. Besides, the preference for room quality is still high for North American people (0.317). Most interestingly, service quality is the feature which has heavy effect on decision making process of people from all regions when traveling with family, particularly for North American and Oceania people. 
TABLE IV: Shapley values of Hotel Features rated by different Groups of Travelers

\begin{tabular}{|c|c|c|c|c|c|c|c|}
\hline \multirow{2}{*}{ Travel Type } & \multirow{2}{*}{ Region } & \multicolumn{6}{|c|}{ Hotel Features } \\
\hline & & Value & Location & Sleep & Room & Cleanliness & Service \\
\hline \multirow{4}{*}{ Business } & Asia & 0.250 & 0.042 & 0.108 & 0.258 & 0.142 & 0.200 \\
\hline & Europe & 0.192 & 0.050 & 0.125 & 0.300 & 0.067 & 0.267 \\
\hline & North America & 0.267 & 0.150 & 0.233 & 0.100 & 0.067 & 0.183 \\
\hline & Oceania & 0.017 & 0.067 & 0.117 & 0.317 & 0.117 & 0.367 \\
\hline \multirow{4}{*}{ Couple } & Asia & 0.265 & 0.117 & 0.158 & 0.153 & 0.153 & 0.154 \\
\hline & Europe & 0.225 & 0.075 & 0.125 & 0.158 & 0.142 & 0.275 \\
\hline & North America & 0.150 & 0.067 & 0.114 & 0.303 & 0.150 & 0.217 \\
\hline & Oceania & 0.100 & 0.100 & 0.183 & 0.217 & 0.133 & 0.267 \\
\hline \multirow{4}{*}{ Family } & Asia & 0.217 & 0.133 & 0.067 & 0.167 & 0.167 & 0.250 \\
\hline & Europe & 0.142 & 0.125 & 0.167 & 0.233 & 0.117 & 0.217 \\
\hline & North America & 0.092 & 0.092 & 0.042 & 0.317 & 0.117 & 0.342 \\
\hline & Oceania & 0.100 & 0.203 & 0.119 & 0.092 & 0.186 & 0.300 \\
\hline
\end{tabular}

\section{Discussion}

The above analysis of Shapley values and interaction indices have demonstrated that the presented fuzzy decision support system was able to provide hotel managers with a better understanding on travelers profiles and their preferences of hotel features. More specifically, they are able to create better business planning to improve hotel services by evaluating the importance of hotel features. Marketing strategy could be also improved with the profile construction for different travel groups from different regions. Consumer behavior was clearly understood through interaction index analysis of hotel features for people traveling with different purposes such as for business or for holiday with partner or family.

In view of sentiment mining application, this paper have considered the feature based sentiment mining under a new perspective which has not been implemented in existing sentiment systems. The presented fuzzy decision support method can provide more utility for feature based summary system in supporting organizations, companies and business managers in decision making process. The output result is easy to interpret and understand, and can thus be used directly by non-technical users. As this is general technique for modeling MCDM process, it can be applied into many areas such as retail industry for customer preferences about products, education industry for student preferences about course contents, politic for understanding the demand of people in society, etc. The presented study case for Hotels in Singapore has demonstrated its effectiveness in Tourism industry. Note that, although the assumption of this work is that the rating of opinion for each aspect should be available for review comments as on TripAdvisor web site. Such information can be mined directly from review text by applying other existing sentiment mining techniques when it is not already available. Therefore, the application of this method is not limited, and can be intergraded widely in most application of sentiment mining.

In view of fuzzy system area, this paper have demonstrated the usefulness of shapley value and interaction index with a real life application of tourism industry. Since, the presented aggregation function uses Choquet Integral which is able take into account the interplay of non-independent criteria and model the interaction between criteria. The MCDM process of consumers can be modeled more accurately and closely to real life situation.

\section{Conclusions}

Due to the growth of internet technology and development of the Web 2.0, online environment has become an important channel for people around the world to seek and share opinions. This has presented a potential gold mine for businesses to construct profiles of target customers that support for their business planning process. Feature based sentiment mining has appeared to be a promising area to help business managers achieve such goals. However, existing summary systems were mainly designed to support for opinion seeking purpose under the perspective of individual users rather than organizations. Namely, existing summary systems were not focusing on answering questions such as what are the key product features which are concerned most by people?, and what are the preferences of people from different backgrounds?. Whereas, such information is particularly important for business managers to improve their products and create better marketing strategies.

In this paper, we have tackled those shortcomings of feature based sentiment summary systems by introducing a relatively new fuzzy decision support method which can model effectively the MCDM process of consumers. This is also the first attempt to explore the usage of shapley value and interaction index in application to Sentiment Mining area. This method was demonstrated to be better than other well-known techniques such as the weighted arithmetic mean and the ordered weighted averaging, since the use of Choquet Integral and fuzzy measure is not only able to model more accurately the nature of MDCM but also measure the interaction between criteria. With a case study for Hotel industry in Singapore, the result analysis have explored the advantages of this technique.

A natural extension of this work is to apply this technique into other areas (retail, education, politics, etc.) and take into account more aspects of discussed features in reviews. In view of sentiment mining application, online sentiment summary systems for business managers could also be developed to extend the benefits of sentiment mining in supporting decision making process for wider range of users. 


\section{REFERENCES}

[1] E. Qualman, Socialnomics: How Social Media Transforms the Way We Live and Do Business. John Wiley and Sons, Inc., Hoboken, New Jersey, 2009.

[2] J. Rong, H. Q. Vu, R. Law, and G. Li, "A behavioral analysis of web sharers and browsers in hong kong using targeted association rule mining," Tourism Management, 2011, in Press.

[3] B. Liu, Handbook of Natural Language Processing. Taylor and Francis Group, LLC, 2010, ch. 11 - Sentiment Analysis and Subjectivity.

[4] M. Hu and B. Liu, "Mining and summarizing customer reviews," in Proceedings of the tenth ACM SIGKDD international conference on Knowledge discovery and data mining, Seattle, WA, USA, August 2004, pp. 168-177.

[5] J.-Y. Yang, H.-J. Kim, and S.-G. Lee, "Feature-based product review summarization utilizing user score," Journal of Information Science and Engineering, vol. 26, pp. 1973-1990, 2010.

[6] K. Shimada and T. Endo, "Seeing several stars : A rating inference task for a document containing several," in Proceedings of the 12th PacificAsia conference on Advances in knowledge discovery and data mining, Osaka, Japan, May 2008, pp. 1006-1014.

[7] J. Ma, J. Lu, and G. Zhang, "Decider: A fuzzy multi-criteria group decision support system," Journal of Knowledge-Based Systems, vol. 23, no. 1, pp. 23-31, Feb. 2010.

[8] P.-C. Chang, C.-H. Liu, and Y.-W. Wang, "A hybrid model by clustering and evolving fuzzy rules for sales decision supports in printed circuit board industry," Journal of Decision Support Systems, vol. 42, no. 3, pp. 1254-1269, Dec. 2006.

[9] E. Ngai and F. Wat, "Fuzzy decision support system for risk analysis in e-commerce development," Decision Support Systems, vol. 40(2), no. 2, pp. 235-255, Aug. 2005.

[10] M. Grabisch and M. Roubens, Application of the Choquet Integral in Multicriteria Decision Making, M. Grabisch, T. Murofushi, and M. Sugeno, Eds. Physica Verlag, 2000.

[11] J. Lu, X. Deng, P. Vroman, X. Zeng, J. Ma, and G. . Zhang, "A fuzzy multi-criteria group decision support system for nonwoven based cosmetic product development evaluation," in IEEE International Conference on Fuzzy Systems, Hong Kong, China, June 2008, pp. 17001707.

[12] F. J. J. Santos and H. A. Camargo, "Fuzzy systems for multicriteria decision making," Clei Electronic Journal, vol. 13, no. 4, p. Paper 4, 2010.

[13] X. Deng, P. Vroman, X. Zeng, and B. Laouisset, "Intelligent decision support tools for multicriteria product design," in Proceeding of IEEE International Conference on Systems Man and Cybernetics, Istanbul, Octorber 2010, pp. 1223-1230.

[14] A. A. Bazzari, M. Osanloo, and B. Karimi, "A new fuzzy multi-criteria decision making model for open pit mines equipment selection," AsiaPacific Journal of Operational Research, vol. 28, no. 3, pp. 279-300, 2011.

[15] G. Beliakov, A. Pradera, and T. Calvo, Aggregation Functions: A Guidefor Practitioners. Springer,Heidelberg, 2007.

[16] G. Beliakov. (2007) fmtools package, version 1.0. http://www.deakin.edu.au/ gleb/aotool.html.

[17] A. Balahur and A. Montoyo, "A feature dependent method for opinion mining and classification," in Proceeding of International Conference on Natural Language Processing and Knowledge Engineering, Beijing, China, Octorber 2008, pp. 1-7.

[18] M. Abulaish, M. N. Doja, and T. Ahmad, "Feature and opinion mining for customer review summarization," Lecture Notes in Computer Science, vol. 5909, pp. 219-224, 2009.

[19] Q. Miao, Q. Li, and D. Zeng, "Fine-grained opinion mining by integrating multiple review sources," Journal of the American Society for Information Science, vol. 61, no. 11, pp. 2288-2299, 2010.

[20] M. Gamon and A. Aue, "Automatic identification of sentiment vocabulary exploiting low association with known sentiment terms," in Proceedings of the ACL Workshop on Feature Engineering for Machine Learning in Natural Language Processing, Michigan, USA, June 2005, pp. 57-64.

[21] X. Fei, H. Wang, and J. Zhu, "Sentiment word identification using the maximum entropy model," in Proceeding of International Conference on Natural Language Processing and Knowledge Engineering, Beijing , China, August 2010, pp. 1-4.
[22] X. Ding, B. Liu, and P. Yu, "A holistic lexicon-based approach to opinion mining," in Proceedings of the international conference on Web search and web data mining, California, United States, February 2008, pp. 231240.

[23] J. Zhu, H. Wang, B. K. Tsou, and M. Zhu, "Multi-aspect opinion polling from textual reviews," in Proceeding of the 18th ACM conference on Information and knowledge management, Hong Kong, China, November 2009, pp. 1799-1802.

[24] T. T. Thet, J.-c. Na, and C. S. G. K. Khoo, "Aspect-based sentiment analysis of movie reviews on discussion boards," Journal of Information Science, vol. 36, no. 6, pp. 823-848, 2010.

[25] S. Hariharan, R. Srimathi, M. Sivasubramanian, and S. Pavithra, "Opinion mining and summarization of reviews in web forums," in Proceedings of the Third Annual ACM Bangalore Conference, Bangalore, India, January 2010, pp. 10-13.

[26] Y. Lu, C. Zhai, and N. Sundaresan, "Rated aspect summarization of short comments," in Proceedings of the 18th international conference on World wide web, Madrid, Spain, April 2009, pp. 131-140.

[27] H. Wang, Y. Lu, and C. Zhai, "Latent aspect rating analysis on review text data : A rating regression approach," in Proceedings of the 16th ACM SIGKDD international conference on Knowledge discovery and data mining, Washington, DC, USA, July 2010, pp. 783-792.

[28] H. Chen and D. Zimbra, "AI and Opinion Mining," IEEE Intelligent Systems, vol. 25, no. 3, pp. 74-80, May 2010.

[29] A. Huettner and P. Subasic, "Fuzzy typing for document management," in Proceedings of the ACL-2000 Conference, Hong Kong, China, Octorber 2000, pp. 26-27.

[30] P. Subasic and A. Heuttner, "Affect analysis of text using fuzzy semantic typing," IEEE Transaction on Fuzzy Systems, vol. 9, no. 4, pp. 483-496, 2011.

[31] S. Nadali, M. A. A. Murad, and R. A. Kadir, "Sentiment classification of customer reviews based on fuzzy logic," in Proceeding of International Symposium in Information Technology, Kuala Lumpur, Malaysia, June 2010, pp. 1037-1044.

[32] C. W.-k. Leung, S. C.-f. Chan, F.-1. Chung, and G. Ngai, "Aprobabilistic rating inference framework formining user preferences from reviews," World Wide Web, vol. 14, pp. 187-215, 2011.

[33] X. Ding and B. Liu, "The utility of linguistic rules in opinion mining," in Proceedings of the 30th annual international ACM SIGIR conference on Research and development in information retrieval, Amsterdam, The Netherlands., July 2007, pp. 811-812.

[34] G. Li and F. Liu, "A clustering-based approach on sentiment analysis," in Procceding of International Conference on Intelligent Systems and Knowledge Engineering, Hangzhou, China, November 2010, pp. 331337.

[35] R. R. Yager, "On ordered weighted averaging aggregation operators in multi-criteria decision making," IEEE Transactions on Systems, Man and Cybernetics, vol. 18, pp. 182-190, 1998.

[36] V. Torra and Y. Narukawa, Modeling Decision. Information Fusion and Aggregation Operators, D. M. Gabbay, Ed. Springer, Berlin, Heidelberg, 2007.

[37] - "The h-index and the number of citations : two fuzzy integrals," IEEE Transactions onFuzzy Systems, vol. 16, no. 3, pp. 795-797, 2008.

[38] G. Beliakov, "Construction of aggregation functions from data using linear programming," Fuzzy Sets and Systems, vol. 160, pp. 65-75, 2009.

[39] P. Meyer and M. Roubens, "On the use of the choquet integral with fuzzy numbers in multiple criteria decision support," Journal of Fuzzy Sets and Systems, vol. 157, no. 7, pp. 927-938, 2006.

[40] G. Beliakov and S. James, "Citation-based journal ranks: The use of fuzzy measures," Journal of Fuzzy Sets and Systems, vol. 167, pp. 101119, 2011.

[41] S. Blair-goldensohn, N. York, K. Hannan, N. York, R. Mcdonald, T. Neylon, G. A. Reis, and J. Reynar, "Building a sentiment summarizer for local service reviews," in Proceeding of WWW 2008 Workshop NLP Challenges in the Information Explosion Era, Beijing, China, April 2008.

[42] J. Khadaroo and B. Seetanah, "The role of transport infrastructure in international tourism development: A gravity model approach," Tourism Management, vol. 29, pp. 831-840, 2008. 